\title{
Accuracy of High-Altitude Photogrammetric Point Clouds in Mapping
}

\section{Tanhuanpaa, Topi}

Springer International Publishing AG 2017

Tanhuanpaa , T , Saarinen , N , Kankare , V , Nurminen , K, Vastaranta , M , Honkavaara , E , Karjalainen , M , Yu , X , Holopainen , M \& Hyyppa , J 2017 , Accuracy of High-Altitude Photogrammetric Point Clouds in Mapping . in I Ivan , A Singleton, J Horak \& T Inspektor (eds), The Rise of Big Spatial Data . Lecture Notes in Geoinformation and Cartography , Springer International Publishing AG , pp. 167-181, GIS Symposium on the Rise of Big Spatial Data , Ostrava , Czech Republic , 16/03/2016 . https://doi.org/10.1007/978-3-319-45123-7_13

http://hdl.handle.net/10138/308009

https://doi.org/10.1007/978-3-319-45123-7_13

unspecified

acceptedVersion

Downloaded from Helda, University of Helsinki institutional repository.

This is an electronic reprint of the original article.

This reprint may differ from the original in pagination and typographic detail.

Please cite the original version. 


\section{ACCURACY OF HIGH-ALTITUDE PHOTOGRAM METRIC POINT CLOUDS IN MAPPING INDIVIDUAL TREE CROWNS}

Topi, Tanhuanpää1,3*; Ninni, Saarinen ${ }^{1,3}$; Ville, Kankare ${ }^{1,3}$; Kimmo, Nurminen2; Mikko, Vastaranta1,3; Eija, Honkavaara2; M ika, Karjalainen 2,3; Xiaowei, Yu²,3; M arkus, Holopainen ${ }^{1,3}$; Juha, Hyyppä2,3.

${ }^{1}$ Department of Forest Sciences, Faculty of Agriculture and Forestry, University of Helsinki, P.O. Box 27 (Latokartanonkaari 7), Fl-00014, Helsinki, Finland, first.last@ helsinki.fi

${ }^{2}$ Finnish Geospatial Research Institute, National Land Survey, Geodeetinrinne 2, Fl-04310 Masala, Finland, first.last@nls.fi

${ }^{3}$ Centre of Excellence in Laser Scanning Research, Finnish Geospatial Research Institute, FI-02431 Masala, Finland

\section{Pre-print of published version}

\section{Reference:}

Tanhuanpää, T., Saarinen, N., Kankare, V., Nurminen, K., Vastaranta, M., Honkavaara, E., Karjalainen, M., Yu, X., Holopainen, M. \& Hyyppä, J. (2017). Accuracy of HighAltitude Photogrammetric Point Clouds in Mapping. In The Rise of Big Spatial Data (pp. 167-181). Springer International Publishing.

DOI: 10.1007/978-3-319-45123-7_13

\section{Disclaimer:}

The PDF document is a copy of the final version of this manuscript that was subsequently accepted by the journal for publication. The paper has been through peer review, but it has not been subject to any additional copy - editing or journal specific formatting (so will look different from the final version of record, which may be accessed following the DOI above depending on your access situation).

\footnotetext{
Abstract

During the past decade, airborne laser scanning (ALS) has established its status as the state-of-the-art method for detailed forest mapping and monitoring. ALS-based methods are widely used in operational forest inventory. Recent advances in the sensor technology and image processing have enabled extraction of dense point clouds also from digital stereo imagery (DSI). Comparing to ALS data, the DSI-based data are cheap and the point cloud densities can easily reach that of ALS. In terms of point density, even the high-altitude DSIbased point clouds would be sufficient for detecting individual tree crowns. However, there are significant differences in the characteristics of ALS and DSI point clouds that likely affect the accuracy of tree detection. In this study, the performance of high-altitude DSI point clouds was compared to low-density ALS in detecting individual trees. The trees were extracted from DSI- and ALS-based canopy height models (CHM) using
} 
watershed segmentation. The use of both smoothed and unsmoothed CHM s was tested. The results show that, even though the spatial resolution of the DSI-based CHM was better, in terms of detecting the trees and the accuracy of height estimates, the low-density ALS performed better. However, utilizing DSI with shorter ground sample distance (GSD) and more suitable image matching algorithms would likely enhance the accuracy of DSI-based approach.

Keywords: image-based point clouds, LiDAR, height models, tree detection, forest

\section{Introduction}

Detailed and spatially accurate datasets form the core of operative forest management. In order to be allocated correctly in both spatial and temporal sense, various management actions require precise knowledge on forest structure. Airborne laser scanning (ALS) has been the latest significant breakthrough in operational forest resource assessment and management. ALS technology, also referred as airborne LiDAR, combines accurate distance measurements with laser light, positioning with global navigation satellite system (GNSS) and precise orientation of the measurement unit with an inertial measurement unit (IMU) (Wehr \& Lohr, 1999). The methodology enables forming spatially accurate three dimensional (3D) point clouds that represent the shape and structure of the scanned object. In forest environment the point clouds have proven to be an accurate means for assessing the vertical structure of the forest canopy (e.g., Lim et al. (2003)). Two distinct methodologies can be distinguished for assessing forest attributes from ALS data. In the so-called area-based approach (ABA), various statistical features describing height distribution of the ALS point cloud are calculated in cell-wise manner for a forest area. The features are utilized in developing e.g., regression models (Næsset, 2002) together with information from field plots for predicting forest inventory attributes for unmeasured grid cells. Typically, low-density ALS data $(<1$ point/m2) are utilized in ABA. An alternative approach utilizing more dense point clouds is referred as individual tree detection (ITD). The methodology is based on identifying the points from the point clouds representing each individual tree crown, calculating tree-level ALS features, and generating tree attributes for the detected trees (Hyyppä \& Inkinen, 1999). The partitioning of the point clouds can be done, e.g., with watershed segmentation also referred as pouring algorithm (Koch et al., 2006), which utilizes canopy height models (CHM), derived from the point clouds. The method seeks the local maxima from the CHM and treats them as seed pixels, i.e., tree tops. The crown area is formed by adding neighboring pixels to the seed as far as their height value is lower than the last added pixel. The CHM is often smoothed prior to the segmentation process in order to decrease the amount of noise in the data. ITD generally requires denser ALS data than ABA ( $>5$ points $/ \mathrm{m} 2$ ). However, several studies have proven that the structure of the forest and the method used for delineation affect the ITD accuracy more than the point density (Kaartinen et al., 2012; Vauhkonen et al., 2012). The price of the ALS datasets increases as the point density gets higher.

Aerial images and photogrammetry have a long tradition in assessing of forest resources. Image-based 3D measurements, concerning, e.g., tree height, have been studied from 1940's. (see, Korpela (2004)). With means of stereophotogrammetry, detecting a common point (e.g., a tree top) from at least two images enables defining its 3D coordinates (XYZ). During recent years, aerial imagery has been widely utilized especially in tree species interpretation, due to its sufficient spatial and spectral resolution (e.g., B. Yu et al. (1999); Held et al. (2003)). However, advances in sensor technology and image processing have enabled generating dense digital stereo imagery (DSI)-based point clouds that offer the same levels of point density as ALS. The DSI-based point clouds are generated with automatic image matching algorithms, e.g., semiglobal matching (Hirschmüller, 2008). The density of the final point cloud depends on the image resolution and the matching algorithm used (White et al., 2013). In case of forestry, the DSI-based point clouds can be 
utilized in, e.g., predicting forest attributes in the same manner as ALS-based point clouds (Nurminen et al., 2013).

In terms of forest resource assessment, both ALS and DSI -based data collection have their strengths and weaknesses. Although highly dependent on the densities, the cost of DSI data is approximately from one third to half of the cost of ALS data (Holopainen et al., 2014; White et al., 2013). Considering the spatial resolution (XY), a given point cloud density can be achieved from higher flying altitude when using DSI, which greatly affects the cost of the data. Also, DSI have much better spectral resolution which can be utilized together with the extracted point cloud, e.g., in species classification. However, the DSI-based point clouds can only cover the upper parts of the canopy that are visible in the images, whereas ALS penetrates the canopy to some extent, reaching the suppressed tree crowns and the ground (Ackermann, 1999). In order to achieve reliable estimates on tree height, the ground elevation has to be known accurately. The current operational forest inventory systems commonly use ALS data for generating the digital terrain model (DTM) and the three dimensional (3D) structure of the trees, whereas DSI are often used only for species interpretation. However, the recent developments, especially in image processing, have made it possible to achieve sufficient point clouds for ABA, using only DSI and existing DTMs (Järnstedt et al., 2012). Also, considering only the point densities of the image-based point clouds, even ITD should be possible using solely materials derived from DSI. Recent studies have shown the differences between the two types of point clouds (Vastaranta et al., 2013; White et al., 2015), but the DSI-based data have rarely been utilized in the level of single trees, i.e., in ITD.

The aim of this study was to investigate the capability of high-altitude DSI-based point clouds in detecting single tree crowns in mature boreal forest. The performance of DSI-based was evaluated by the number of correctly matched field measured reference trees, the accuracy of individual height measurements, and the correctness of the overall plot-level height distributions. For comparison, the same procedures were also applied to CHM s derived from low-density ALS data.

\section{Materials and methods}

\section{Study area}

The study area of $5 \times 5-\mathrm{km}$ is located in Evo, southern Finland (61.19N, 25.11트) (Figure 1). It belongs to the southern Boreal Forest Zone and contains approximately 2000 ha of managed boreal forest. The average stand size is slightly less than 1 ha. The area consists of a mixture of forest stands, varying from natural to intensively managed forests. The elevation of the area varies from $125 \mathrm{~m}$ to $185 \mathrm{~m}$ above sea level. The dominant tree species are Scots pine (Pinus sylvestris L.) and Norway spruce (Picea abies (L.) H. Karst) contributing $40 \%$ and $35 \%$ of the total volume, respectively. The share of all deciduous trees together is $24 \%$ of the total volume. 


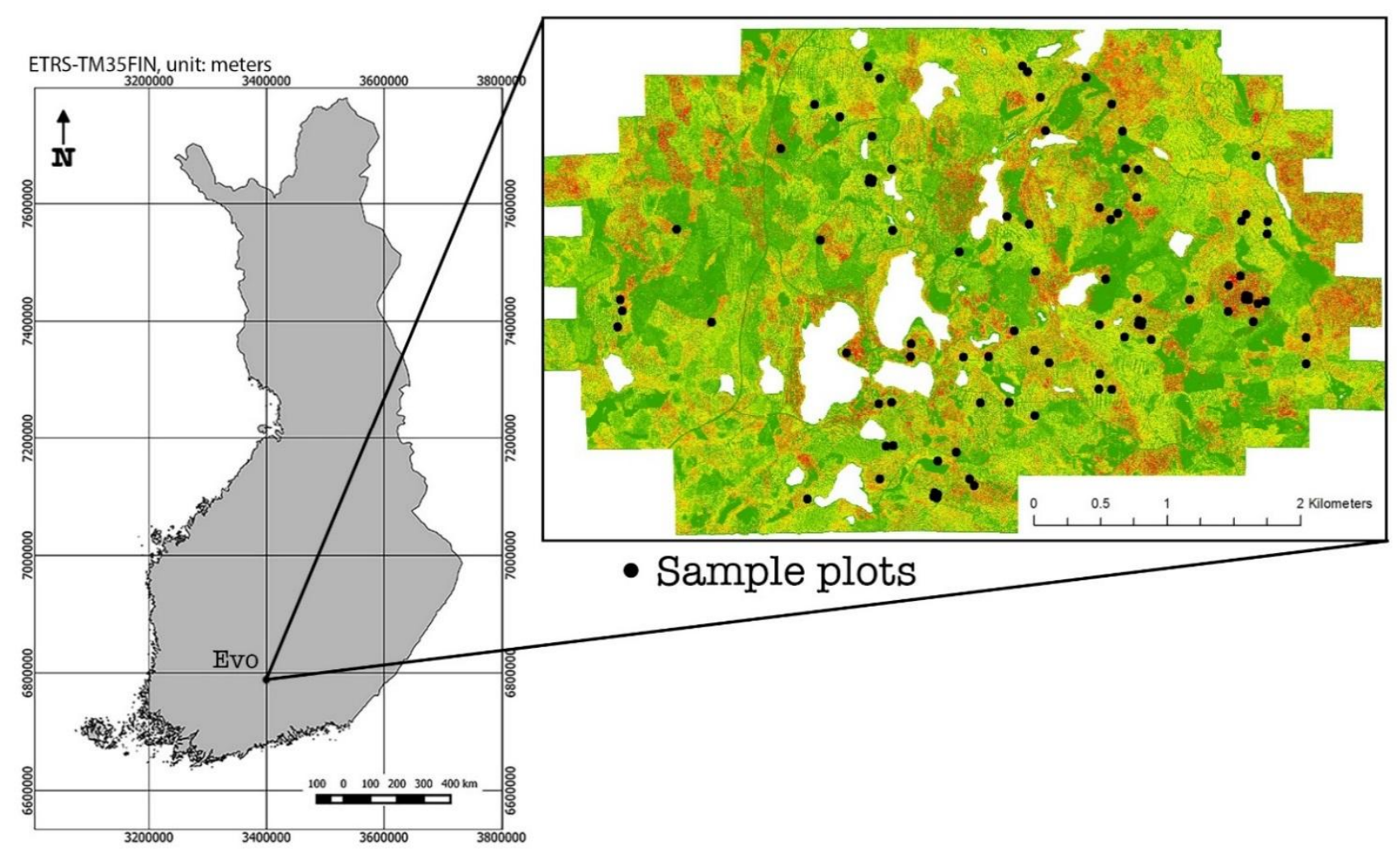

Fig. 1. The study area: Black dots in the right-hand side indicate the location of the field plots.

Field data

The field data, consisting of 91 plots ( $32 \mathrm{~m} \times 32 \mathrm{~m}$ ), was collected in summer 2014. A tree map was generated for all plots using terrestrial laser scanner. The tree maps were validated in the field: the trees missing from the maps were added to it and incorrect (i.e., non-existing) trees were removed from the map. After the validation, all trees with diameter-at-breast-height (DBH) over $5 \mathrm{~cm}$ were measured manually. DBH was measured with steel caliper and the tree heights with electronic hypsometer.

The locations of the individual trees were brought into common coordinate system by determining the position of each sample plot. The locations were calculated using the geographic coordinates of the plot centers and four corners. Plot center positions were measured using differential GPS (Leica GPS 120, Switzerland) with sub-meter accuracy. A total station (Leica TS02, Switzerland) was used to measure the exact distance to each plot center. Plot position was further adjusted manually using ALS data. In this procedure, the locations of all trees on the tree map were used to help find the true location of the plot in the ALS point cloud. The plot was shifted and rotated so that the tree locations within the map aligned properly with the point cloud.

For this study only the mature plots (i.e., the plots with basal area-weighted DBH over $26 \mathrm{~cm}$ ) were included in this study. This subset of 39 plots was further divided into sub-groups by the dominant tree species, contributing over $70 \%$ of the basal area. The classes were defined as described in Table 1. From the 39 mature plots, only the trees with commercial value were taken into consideration. Hence the trees with height under 14 meters were excluded from the data. Altogether, there were 1684 trees in the final field reference. The descriptive statistics of all sub-groups are presented in Table 2. 
Table 1. Definitions of study subgroups.

\begin{tabular}{l|l|l} 
Sub-group & Group description & $\mathbf{n}$ \\
\hline Pine & Plots with pine representing over $70 \%$ of the basal area & 12 \\
Spruce & Plots with spruce representing over $70 \%$ of the basal area & 15 \\
Mixed & Mixed plots with none of the species representing over $70 \%$ of the basal area & 12 \\
Total & All plots & 39
\end{tabular}

Table 2. The descriptive statistics of 39 sample plots of $32 \times 32$ m used in this study.

\begin{tabular}{|c|c|c|c|c|c|}
\hline & & Minimum & Maximum & Mean & St. Dev. \\
\hline \multirow[t]{5}{*}{ Pine } & M ean height (m) & 21.4 & 32.1 & 25.8 & 3.6 \\
\hline & M ean DBH (cm) & 26.3 & 46.4 & 30.8 & 6.0 \\
\hline & Basal area (m2/ha) & 17.3 & 40.3 & 26.7 & 7.1 \\
\hline & Volume (m3/ha) & 164.5 & 518.4 & 300.0 & 109.4 \\
\hline & Plot density (trees/ha) & 391 & 1035 & 565 & 201 \\
\hline \multirow[t]{5}{*}{ Spruce } & M ean height (m) & 25.4 & 33.4 & 29.2 & 2.6 \\
\hline & M ean DBH (cm) & 26.0 & 42.1 & 33.9 & 5.4 \\
\hline & Basal area (m2/ha) & 22.1 & 38.9 & 32.8 & 5.1 \\
\hline & Volume (m3/ha) & 242.6 & 484.9 & 390.8 & 75.0 \\
\hline & Plot density (trees/ha) & 342 & 879 & 585 & 159 \\
\hline \multirow[t]{5}{*}{ Mixed } & M ean height (m) & 23.1 & 31.6 & 27.4 & 2.7 \\
\hline & M ean DBH $(\mathrm{cm})$ & 26.6 & 41.6 & 33.4 & 5.0 \\
\hline & Basal area (m2/ha) & 15.2 & 43.2 & 33.1 & 8.0 \\
\hline & Volume (m3/ha) & 177.7 & 508.2 & 349.1 & 96.4 \\
\hline & Plot density (trees/ha) & 342 & 2217 & 909 & 482 \\
\hline \multirow[t]{5}{*}{ Total } & M ean height (m) & 21.4 & 33.4 & 27.6 & 3.2 \\
\hline & M ean DBH (cm) & 26.0 & 46.4 & 32.8 & 5.5 \\
\hline & Basal area (m2/ha) & 15.2 & 43.2 & 31.0 & 7.2 \\
\hline & Volume (m3/ha) & 164.5 & 518.4 & 350.0 & 98.4 \\
\hline & Plot density (trees/ha) & 342 & 2217 & 678 & 335 \\
\hline
\end{tabular}

Aerial images

The aerial images were acquired on the 22nd of M ay 2014 with a Z/I Imaging DM C (Digital M apping Camera) photogrammetric aerial camera. The image block consisted of two flying strips of 12 images. The forward and 
side overlap of the pictures were $80 \%$ and $64 \%$, respectively. The images were acquired from altitude of approximately $5000 \mathrm{~m}$ above the mean ground level which lead to $50 \mathrm{~cm}$ ground sample distance (GSD). The width of an image strip was $6.9 \mathrm{~km}$, and the distance between adjacent flight lines was $2.5 \mathrm{~km}$. The image orientation was done in BAE Systems Socet Set software (San Diego, California, USA) on the basis of exterior orientation values. The final orientation based on automatic tie points and 40 interactively measured ground control points. The ground control points were derived from elevation model and orthophotos provided by Finnish National Land Survey (NLS). Three radial distortion parameters were solved with on-the-job calibration. The root mean square error (RM SE) values of the adjustment were $0.266 \mathrm{~m}(\mathrm{X}), 0.400 \mathrm{~m}(\mathrm{Y})$ and $1.187 \mathrm{~m}$ (Z).

The calculation of stereo models was carried out with NGATE (Next Generation Automatic Terrain Extraction) module of the Socet Set software bundle using an altered strategy file for forestry applications. Extraction of digital surface model (DSM) was done for each stereo model of consecutive images in the same strip, but inter-strip stereo models were not used in the calculation. National DTM was applied to normalize DSM into $\mathrm{CHM}$. The national DTM was derived from ALS data and had resolution of $2 \mathrm{~m}$. The image-based CHM is referred as $\mathrm{CHM}$ image.

ALS data

ALS data were acquired in late M ay 2014, using a Leica ALS70-HA SN7202 system (Leica Geosystems AG, Heerbrugg, Switzerland) operating at a pulse rate of $105 \mathrm{kHz}$. Flying altitude was $2500 \mathrm{~m}$ above sea level and flying speed 150 knots. On average, the measurement density was 0.7 pulses $/ \mathrm{m} 2$. The system was configured to record up to five echoes per pulse, i.e. first or only, last and 1 to 3 intermediates. Hence, the point density of the ALS point clouds was approximately 1-4 points/m2 depending on the vegetation structure.

The ALS data were processed with Terra Scan software (Terrasolid, Helsinki, Finland) as follows. First, the point cloud density and pattern was unified by minimizing the flightline overlap. After this, all water surfaces were masked out from the data using water boundaries. Also, all points under ground level were also removed from the data with classification tools of TerraScan. The ground points were classified with tools based on methods by Axelsson (2000). The digital surface model (DSM ) was created from all vegetation points with resolution of $1 \mathrm{~m}$. The CHM was obtained by subtracting the national DTM from the created DSM. The ALS-based CHM is referred as CHM als.

\section{Tree delineation}

Individual crown segments were delineated from the CHM s with watershed segmentation process (see e.g., Pitkänen et al. (2004); Koch et al. (2006)). Both smoothed and unsmoothed CHMs were tested for tree extraction. A simple $3 \times 3$ pixel moving average filter was used for the smoothing process. The unsmoothed CHM s derived from aerial images and ALS data were denoted CHM image and CHM als. The smoothed CHM S were denoted CHM image_smooth and CHM als_smooth, respectively.

The height and location of the crown segments were adopted from the maximum value of the unsmoothed CHM (i.e., CHM image and CHMals) inside each segment. If the CHM maximum was a "plateau" consisting from several pixels, the location was determined as the mean location of the plateau pixels. The final tree candidates were chosen among the crown segments in terms of tree height. Focusing on mature trees, only crown segments with $\mathrm{CHM}$ value (i.e., tree height) of at least 14 meters were considered to represent actual trees.

\section{Tree matching}

For assessing the tree-level accuracy of the tree detection procedure, the tree candidates were matched to field measured reference trees with the method described in Kaartinen et al. (2012). The procedure matches 
location points of reference trees and tree candidates that are most alike in terms of location. Tree candidates that were more than 5 meters from reference trees were automatically treated as undetected. The matching started by determining a distance matrix that consisted of distances between every reference tree and tree candidate. The first reference tree to be matched was the one with the nearest tree candidate (shortest distance in the distance matrix). After the match was made, both the reference tree and the matched tree candidate were removed from the distance matrix. The procedure was repeated until there were no tree candidates left within $5 \mathrm{~m}$ of the remaining reference trees.

Accuracy assessment

The accuracy resulted from using either CHM image, CHM als, CHM image_smooth, or CHMals_smooth, was estimated through six different measures: percentage of matched trees (Eq. 1.), relative error of omission and commission (Eq. 2 and 3.), root mean square error (RM SE) and bias of the predicted height of matched trees (Eq. 4. and 5.). In addition, the correctness of the plot-level height distributions was evaluated with Reynolds error index (Reynolds et al., 1988) (El, Eq. 6.) and relative error index (Packalén \& Maltamo, 2008) (Elrel, Eq. 7.). El shows the absolute difference between distributions whereas Elrel shows the difference in relation to the total number of observations. The height distributions were created from the CHM-derived heights using $1 \mathrm{~m}$ bin size. All measures were calculated separately for all plots, pine and spruce dominated plots, and mixed plots.

Matched trees $S_{\text {rel }}=\frac{N_{\text {matched }}}{N_{\text {ref }}}$,

Error of omission $_{\text {rel }}=\frac{N_{\text {missed }}}{N_{\text {ref }}}$

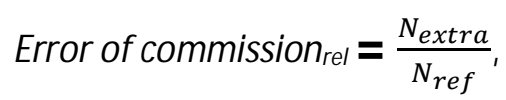

where $\mathrm{N}_{\text {matched }}$ is the number of successfully matched trees, $\mathrm{N}_{\text {missed }}$ the number of the undetected trees, $\mathrm{N}_{\text {extra }}$ the number of unmatched tree candidates and $\mathrm{N}_{\text {rel }}$ the number of reference trees.

$\mathrm{RMSE}=\sqrt{\frac{\sum_{i=1}^{n}\left(x_{o b s, i}-x_{\text {model }, i}\right)^{2}}{n}}$

bias $=\frac{\sum_{i=1}^{n}\left(x_{o b s, i}-x_{\text {model }, i}\right)}{n}$,

where $\mathrm{n}$ is the number of trees, $\mathrm{x}_{\mathrm{obs}}$ the field measured tree height $\mathrm{i}$, and $\mathrm{x}_{\text {model }}$ the CHM derived tree height i.

$\mathrm{El}=\sum_{i=1}^{k} w_{i}\left|f_{i}-\hat{f}_{i}\right|$,

$\mathrm{El}_{\mathrm{rel}}=\sum_{i=1}^{k} 0.5\left|\frac{f_{i}}{N}-\frac{\hat{f}_{i}}{\widehat{N}}\right|$,

where $\mathrm{k}$ is the number of height classes, $\mathrm{w}_{\mathrm{i}}$ is the weight of class $\mathrm{i}$, $\mathrm{f}_{\mathrm{i}}$ is the true number of trees in height class $\mathrm{i}, \hat{f}_{i}$ is the predicted number of trees in height class $\mathrm{i}, \mathrm{N}$ is the true number, and $\widehat{N}$ the predicted number of trees on the plot.

To find the optimal procedure for detecting and identifying individual trees within this study, the approaches $\left(\mathrm{CHM}_{\text {image, }} \mathrm{CHM}_{\text {als, }} \mathrm{CHM}_{\text {image } \_ \text {smooth, }}\right.$ or $\left.\mathrm{CHM}_{\text {als_smooth }}\right)$ were ranked according to their performance in terms of the six measures. The ranking was made on basis of the relative values. The best approach in, e.g., pine dominated stands in terms of RM SE received rank 1, the second best rank 2, etc. Thus, the approach with the smallest overall sum of ranks could be argued as the best within the study. Because the number of found 
trees and the error of commission are actually two sides of the same phenomenon, only the latter was taken into consideration when summing up the ranks.

\section{Results and discussion}

When considering the results, the difference in the costs of the DSI- and ALS-based data has to be taken into consideration. Even though the cell size of the image-based CHM s was significantly lower ( $1 \mathrm{~m}$ for ALS and $0.5 \mathrm{~m}$ for DSI), the acquisition of DSI materials was significantly more efficient. For DSI, the whole study area was covered from $5000 \mathrm{~m}$ with two flight lines. Similarly, covering the area by means of ALS from $2500 \mathrm{~m}$ required 4 main flight lines and two lines across the main lines. On the other hand, comparing to ALS data, generating surface models from DSI requires heavy pre-processing (White et al., 2013), which also affects the overall efficiency of the method.

The accuracies concerning the detection and matching of individual trees are presented in Table 3 . The results are reported separately for each approach (i.e., different height models) and sub-group. Columns denoted abs show the absolute number of trees, whereas the relative amounts are reported in rel columns. Column rank shows the rank of each approach within sub-groups.

Table 3. The detection accuracy of watershed segmentation when applied to four different height models. The results are given separately for pine and spruce dominated plots, plots with mixed vegetation, and all plots together.

Found trees

Error of omission

Error of commission

\begin{tabular}{ll|ccc|ccc|ccc} 
& & abs & rel & rank & abs & rel & rank & abs & rel & rank \\
\hline CHM $_{\text {image }}$ & pine & 434 & 0.93 & 1 & 31 & 0.07 & 1 & 362 & 0.46 & 4 \\
& spruce & 564 & 0.85 & 1 & 101 & 0.15 & 1 & 342 & 0.38 & 4 \\
& mixed & 427 & 0.77 & 1 & 127 & 0.23 & 1 & 226 & 0.35 & 4 \\
& total & $\mathbf{1 4 2 5}$ & $\mathbf{0 . 8 5}$ & 1 & $\mathbf{2 5 9}$ & $\mathbf{0 . 1 5}$ & 1 & $\mathbf{9 3 0}$ & $\mathbf{0 . 4 0}$ & 4 \\
\hline CHM $_{\text {image_smooth }}$ & pine & 341 & 0.73 & 3 & 124 & 0.27 & 3 & 87 & 0.20 & 2 \\
& spruce & 348 & 0.52 & 3 & 317 & 0.48 & 3 & 80 & 0.19 & 3 \\
& mixed & 278 & 0.50 & 3 & 276 & 0.50 & 3 & 67 & 0.19 & 3 \\
& total & $\mathbf{9 6 7}$ & $\mathbf{0 . 5 7}$ & 3 & $\mathbf{7 1 7}$ & $\mathbf{0 . 4 3}$ & 3 & $\mathbf{2 3 4}$ & $\mathbf{0 . 2 0}$ & 3 \\
\hline $\mathbf{C H M}_{\text {als }}$ & pine & 400 & 0.86 & 2 & 65 & 0.14 & 2 & 158 & 0.28 & 3 \\
& spruce & 455 & 0.68 & 2 & 210 & 0.32 & 2 & 80 & 0.15 & 2 \\
& mixed & 344 & 0.62 & 2 & 210 & 0.38 & 2 & 60 & 0.15 & 2 \\
& total & $\mathbf{1 1 9 9}$ & $\mathbf{0 . 7 1}$ & 2 & $\mathbf{4 8 5}$ & $\mathbf{0 . 2 9}$ & $\mathbf{2}$ & $\mathbf{2 9 8}$ & $\mathbf{0 . 2 0}$ & 2 \\
\hline $\mathbf{C H M}_{\text {als_smooth }}$ & pine & 233 & 0.50 & 4 & 232 & 0.50 & 4 & 15 & 0.06 & 1 \\
& spruce & 288 & 0.43 & 4 & 377 & 0.57 & 4 & 12 & 0.04 & 1 \\
& mixed & 214 & 0.39 & 4 & 340 & 0.61 & 4 & 12 & 0.05 & 1 \\
& total & $\mathbf{7 3 5}$ & $\mathbf{0 . 4 4}$ & 4 & $\mathbf{9 4 9}$ & $\mathbf{0 . 5 6}$ & 4 & $\mathbf{3 9}$ & $\mathbf{0 . 0 5}$ & 1 \\
& & & & & & & & &
\end{tabular}


Depending on the sub-group and the $\mathrm{CHM}$ used for detecting the trees, the segmentation process identified $39-93 \%$ of the trees. The detection rate was the best when using $\mathrm{CHM}_{\text {image }}$ (unfiltered image-based $\mathrm{CHM}$ ), whereas $\mathrm{CHM}_{\text {als_smooth }}$ resulted the lowest detection rate. Logically, the error of omission follows the same pattern. However, when examining the error of commission, the performance of the approaches changes drastically. Altogether, $4-46 \%$ of the tree candidates produced could not be matched to any reference tree. Here, $\mathrm{CHM}_{\text {als_smooth }}$ gave the most accurate results whereas $\mathrm{CHM}_{\text {image }}$ produced the most extra trees in every sub-group. In terms of the total number of the produced tree candidates (i.e., the number of found trees and the error of commission together), $\mathrm{CHM}_{\text {image }}$ and $\mathrm{CHM}_{\text {als }}$ smooth represent the study extremes. The former produces the largest number of tree candidates (2355) whereas the latter results the smallest number of tree

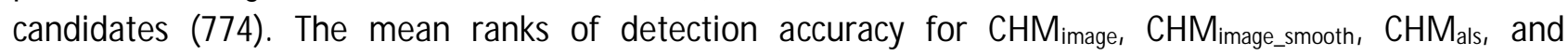
$\mathrm{CHM}_{\text {als_smooth }}$ were $2.5,2.9,2.1$, and 2.5 respectively.

According to the presented mean ranks and within the methods tested, utilizing $\mathrm{CHM}_{\text {als }}$ seems to perform the best in detecting individual tree crowns. Although the resolution of the DSI-based CHM s was higher, the ALS-based CHM s captured the variation of the canopy better. When the noise (i.e., the number of false peaks in the surface) was reduced the number of resulting tree candidates reduced to a significant underestimate. The strong decline in the number of detected trees between $\mathrm{CHM}_{\text {image }}$ and $\mathrm{CHM}_{\text {image_smooth }}$ can result from using too heavy filtering. Using the mean filter seems to smoothen the surface too much as the number of segmentation seed points (i.e., the local maxima) decreases rapidly. The filtering also affects the ALS-based $\mathrm{CHM}$ s. Even though the vertical canopy structure is captured better in $\mathrm{CHM}_{\text {als, }}$ the filtering seems to be too

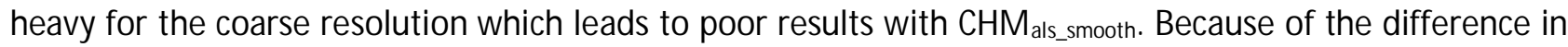
CHM resolutions (i.e., $1 \mathrm{~m}$ for ALS-based CHM s and $0.5 \mathrm{~m}$ for DSI-based CHM s) however, the changes are not directly comparable. Utilizing a different kind of filter for both ALS and DSI-based data might decrease the number of tree candidates but still help to preserve more of the height variation caused from actual tree tops. For example, Hyyppä et al. (2001) utilized a filter weighing the center of the filter window. Still, when compared to previous studies on ALS-based tree detection, also the performance of $\mathrm{CHM}_{\text {image }}$ seems reasonable. In an international comparison of ALS-based detection of individual trees by Kaartinen et al. (2012), the methods utilizing low-density ALS detected $25-90 \%$ of the trees whereas the error of commission was between $0-34.7 \%$.

The results concerning the accuracy of the height measurements on the matched trees and the correctness of height distributions are presented in Table 4. Again, column rank shows the goodness within all CHMs used. In terms of height measurement accuracy, $\mathrm{CHM}_{\text {als }}$ performs the best in nearly all subgroups. For $\mathrm{CHM}_{\text {als, }}$ the RM SE and bias of estimated tree heights were between 0.11-0.16 $\mathrm{m}$ and 0.1-0.5 m, respectively. The mean ranks for the accuracy of tree height for $\mathrm{CHM}_{\text {image, }} \mathrm{CHM}_{\text {image_smooth, }} \mathrm{CHM}_{\text {als, }}$ and $\mathrm{CHM}_{\text {als_smooth }}$ were 2.6, $2.9,1.1$, and 3.4 respectively. 
Table 4. The accuracy of height estimates for the detected trees. The results are given separately for each height model used and for pine and spruce dominated plots, plots with mixed vegetation, and all plots together. Columns denoted abs show the absolute values, whereas the relative values are reported in rel columns. Column El shows the Reynolds error index and Elrel the relative error index.

RM SE of height

bias of height

Error indices

\begin{tabular}{|c|c|c|c|c|c|c|c|c|c|c|}
\hline & & abs (m) & rel (\%) & rank & abs (m) & rel (\%) & rank & $\mathrm{El}$ & $\mathrm{El}_{\mathrm{rel}}$ & rank \\
\hline \multirow{4}{*}{$\mathrm{CHM}_{\text {image }}$} & pine & 4.17 & 0.17 & 2 & 1.12 & 0.05 & 3 & 61 & 0.52 & 4 \\
\hline & spruce & 4.32 & 0.17 & 3 & 0.93 & 0.04 & 2 & 52 & 0.52 & 2 \\
\hline & mixed & 5.36 & 0.23 & 3 & -0.71 & -0.03 & 2 & 50 & 0.48 & 2 \\
\hline & total & 4.61 & 0.23 & 3 & 0.50 & -0.03 & 2 & 54 & 0.49 & 3 \\
\hline \multirow[t]{4}{*}{$\mathrm{CHM}_{\text {image_smooth }}$} & pine & 4.34 & 0.18 & 3 & 0.73 & 0.03 & 1 & 38 & 0.49 & 2 \\
\hline & spruce & 4.65 & 0.18 & 4 & 0.98 & 0.04 & 3 & 36 & 0.52 & 3 \\
\hline & mixed & 5.64 & 0.24 & 4 & -0.82 & -0.03 & 3 & 37 & 0.49 & 3 \\
\hline & total & 4.85 & 0.24 & 4 & 0.37 & -0.03 & 3 & 37 & 0.49 & 2 \\
\hline \multirow[t]{4}{*}{ CHM $_{\text {als }}$} & pine & 2.82 & 0.12 & 1 & 1.10 & 0.05 & 2 & 38 & 0.45 & 1 \\
\hline & spruce & 2.78 & 0.11 & 1 & 0.48 & 0.02 & 1 & 26 & 0.43 & 1 \\
\hline & mixed & 3.82 & 0.16 & 1 & 0.17 & 0.01 & 1 & 34 & 0.42 & 1 \\
\hline & total & 3.13 & 0.16 & 1 & 0.60 & 0.01 & 1 & 32 & 0.40 & 1 \\
\hline \multirow[t]{4}{*}{$\mathrm{CHM}_{\text {als_smooth }}$} & pine & 4.40 & 0.18 & 4 & 2.36 & 0.10 & 4 & 34 & 0.52 & 3 \\
\hline & spruce & 4.42 & 0.16 & 2 & 2.47 & 0.09 & 4 & 34 & 0.55 & 4 \\
\hline & mixed & 4.69 & 0.18 & 2 & 2.17 & 0.09 & 4 & 37 & 0.52 & 4 \\
\hline & total & 4.49 & 0.18 & 2 & 2.35 & 0.09 & 4 & 35 & 0.49 & 4 \\
\hline
\end{tabular}

The tree height was underestimated for nearly all sub-groups with all four methods, which is typical for both DSI- and ALS-based approaches (e.g., St-Onge et al. (2004); Gaveau and Hill (2003)). The sub-group consisting of mixed plots makes an exception. With both DSI-based CHMs, the height of trees on mixed sub-group was overestimated. This suggests, that the DSI-based CHM s were not able to reach the crowns of the suppressed trees in more heterogeneous surroundings. For example, in Vastaranta et al. (2013) it was found that the lower percentiles of DSI-based height distributions were higher than those based on ALS data. When considering the RM SE of the height estimates, the ALS data perform better than DSI in all sub groups. Earlier studies on the relative accuracies of ALS- and DSI-based height measurements in complex terrain are rare. However, individual accuracies of both methods have been widely tested. For example, Korpela (2004) measured individual tree heights from DSI with standard deviation of 0.3-1.0 meters. Similarly, Persson et al. (2002) measured tree heights from ALS data with RM SE of 0.63 meters. In this study, the accuracy of height measurements was considerably lower for both DSI- and ALS-based methods. This is likely resulting from the more coarse resolution of both ALS and DSI datasets used in this study.

$\mathrm{CHM}_{\text {als }}$ performs the best also with this respect to $\mathrm{El}_{\text {rel, }}$, that is actually a combination of the number of tree candidates and the accuracy of their height estimates. However, as El rel varies between 0.40-0.55, 
approximately half of the tree candidates are placed in erroneous height bins. Both missed and extra trees (i.e., error of omission and commission) are included in the figure.

According to the summarizing score in Table $5, \mathrm{CHM}_{\text {als }}$ seems to perform better than the two image-based CHM s in mapping individual tree crowns, whereas $\mathrm{CHM}_{\text {als smooth }}$ performed the worst from the four $\mathrm{CHM} \mathrm{s}$ tested. Even though the spatial resolution of the image-based CHM s was higher than in ALS-based CHM s, they fall behind low-density ALS in the ability of capturing crown-level variation and small openings in the canopy.

Table 5. Total rank score sums (referred as abs) and mean ranks for all four height models.

\section{Total rank score}

\begin{tabular}{l|cc} 
& abs & mean \\
\hline CHM $_{\text {image }}$ & 51 & 2.6 \\
CHM $_{\text {image_smooth }}$ & 58 & 2.9 \\
CHM $_{\text {als }}$ & 30 & 1.5 \\
CHM $_{\text {als_smooth }}$ & 61 & 3.1
\end{tabular}

The relatively high spatial resolution leads to over-segmentation if the image-based CHM is not smoothed, but then again, if a mean filter is used, too much of the variation within the CHM is lost which leads to undersegmentation. This also reflects to the matching results. If the canopy is heavily over-segmented, most of the reference trees can be linked to a tree candidate within the constraints of the algorithm. Contrary, the number of matched trees decreases with under-segmentation because there are not enough tree candidates to match. The problem could be approached through utilizing a more detailed algorithm when matching the tree candidates with the field measured trees. For example, X. Yu et al. (2006) utilized the principle of Hausdorff distance when matching the trees between two subsequent scannings according to their locations, whereas Olofsson et al. (2008), included both tree location and height in the matching procedure. However, in this study we settled for using a very simple matching algorithm when comparing the different approaches.

Considering the ranking on grounds of the five criteria (i.e., the errors of omission and commission, RMSE and bias of height, and Elrel), the method utilizing CHM als seem to perform the best, whereas the second best overall rank was achieved using CHMimge. For both ALS- and DSI-based CHMs, applying a simple 3x3 pixel average filter resulted worse overall results than using the using the unsmoothed CHM. The results indicate that, despite the lower spatial resolution, $\mathrm{CHM}$ als is more capable in detecting small-scale variation in the canopy than CHM image. However, this does not necessarily mean that ALS-based CHM s would be superior to the DSI-based CHM s. With the cost of the low-density ALS, a DSI dataset with much shorter GSD could be acquired. Also, more suitable image matching algorithm could improve the accuracy of DSI-based height models, even with the GSD used in this study.

\section{Conclusions}

In terms of the cost of data acquisition, the high-altitude DSI point clouds seem to offer an efficient means for creating fine-scale CHMs for mapping individual tree crowns. However, the fine resolution does not necessarily provide the same level of accuracy in describing the forest canopy as the ALS-based CHM s of the same resolution. Also, further research is needed for assessing the effect of different image matching algorithms in forest conditions as well as for utilizing DSI with shorter GSD.

\section{Acknowledgement}


This study has been conducted with funding from the European Community's Seventh Framework Programme [FP7/2007-2013] under grant agreement number 606971, Finnish Cultural Foundation under grant 00150939, and from the Academy of Finland in the form of the Centre of Excellence in Laser Scanning Research (project number 272195)

\section{REFERENCES}

Ackermann, F. (1999). Airborne laser scanning-present status and future expectations. ISPRS Journal of Photogrammetry and Remote Sensing, 54(2), 64-67.

Axelsson, P. (2000). DEM generation from laser scanner data using adaptive TIN models. International Archives of Photogrammetry and Remote Sensing, 33(B4/ 1; PART 4), 111-118.

Gaveau, D. L., \& Hill, R. A. (2003). Quantifying canopy height underestimation by laser pulse penetration in small-footprint airborne laser scanning data. Canadian Journal of Remote Sensing, 29(5), 650-657.

Held, A., Ticehurst, C., Lymburner, L., \& Williams, N. (2003). High resolution mapping of tropical mangrove ecosystems using hyperspectral and radar remote sensing. International Journal of Remote Sensing, 24(13), 2739-2759.

Hirschmüller, H. (2008). Stereo processing by semiglobal matching and mutual information. Pattern Analysis and $M$ achine Intelligence, IEEE Transactions on, 30(2), 328-341.

Holopainen, M ., Vastaranta, M., \& Hyyppä, J. (2014). Outlook for the next generation's precision forestry in Finland. Forests, 5(7), 1682-1694.

Hyyppä, J., \& Inkinen, M . (1999). Detecting and estimating attributes for single trees using laser scanner. The photogrammetric journal of Finland, 16(2), 27-42.

Hyyppä, J., Kelle, O., Lehikoinen, M., \& Inkinen, M . (2001). A segmentation-based method to retrieve stem volume estimates from 3-D tree height models produced by laser scanners. Geoscience and Remote Sensing, IEEE Transactions on, 39(5), 969-975.

Järnstedt, J., Pekkarinen, A., Tuominen, S., Ginzler, C., Holopainen, M., \& Viitala, R. (2012). Forest variable estimation using a high-resolution digital surface model. ISPRS Journal of Photogrammetry and Remote Sensing, 74, 78-84.

Kaartinen, H., Hyyppä, J., Yu, X., Vastaranta, M., Hyyppä, H., Kukko, A., . . . Morsdorf, F. (2012). An international comparison of individual tree detection and extraction using airborne laser scanning. Remote Sensing, 4(4), 950-974.

Koch, B., Heyder, U., \& Weinacker, H. (2006). Detection of individual tree crowns in airborne lidar data. Photogrammetric Engineering \& Remote Sensing, 72(4), 357-363.

Korpela, I. (2004). Individual tree measurements by means of digital aerial photogrammetry (Vol. 3): Finnish Society of Forest Science Helsinki, Finland.

Lim, K., Treitz, P., Wulder, M., St-Onge, B., \& Flood, M. (2003). LiDAR remote sensing of forest structure. Progress in physical geography, 27(1), 88-106.

Næsset, E. (2002). Predicting forest stand characteristics with airborne scanning laser using a practical twostage procedure and field data. Remote Sensing of Environment, 80(1), 88-99. 
Nurminen, K., Karjalainen, M., Yu, X., Hyyppä, J., \& Honkavaara, E. (2013). Performance of dense digital surface models based on image matching in the estimation of plot-level forest variables. ISPRS Journal of Photogrammetry and Remote Sensing, 83, 104-115.

Olofsson, K., Lindberg, E., \& Holmgren, J. (2008). A method for linking field-surveyed and aerial-detected single trees using cross correlation of position images and the optimization of weighted tree list graphs. Proceedings of Silvilaser, 2008, 8th.

Packalén, P., \& M altamo, M. (2008). Estimation of species-specific diameter distributions using airborne laser scanning and aerial photographs. Canadian Journal of Forest Research, 38(7), 1750-1760.

Persson, A. , Holmgren, J., \& Söderman, U. (2002). Detecting and measuring individual trees using an airborne laser scanner. Photogrammetric Engineering and Remote Sensing, 68(9), 925-932.

Pitkänen, J., Maltamo, M., Hyyppä, J., \& Yu, X. (2004). Adaptive methods for individual tree detection on airborne laser based canopy height model. International Archives of Photogrammetry, Remote Sensing and Spatial Information Sciences, 36(8), 187-191.

Reynolds, M. R., Burk, T. E., \& Huang, W.-C. (1988). Goodness-of-fit tests and model selection procedures for diameter distribution models. Forest Science, 34(2), 373-399.

St-Onge, B., Jumelet, J., Cobello, M., \& Véga, C. (2004). M easuring individual tree height using a combination of stereophotogrammetry and lidar. Canadian Journal of Forest Research, 34(10), 2122-2130.

Vastaranta, M., Wulder, M. A., White, J. C., Pekkarinen, A., Tuominen, S., Ginzler, C., . . Hyyppä, H. (2013). Airborne laser scanning and digital stereo imagery measures of forest structure: Comparative results and implications to forest mapping and inventory update. Canadian Journal of Remote Sensing, 39(5), 382-395.

Vauhkonen, J., Ene, L., Gupta, S., Heinzel, J., Holmgren, J., Pitkänen, J., . . . Hauglin, K. M. (2012). Comparative testing of single-tree detection algorithms under different types of forest. Forestry, cpr051.

Wehr, A., \& Lohr, U. (1999). Airborne laser scanning-an introduction and overview. ISPRS Journal of Photogrammetry and Remote Sensing, 54(2), 68-82.

White, J. C., Stepper, C., Tompalski, P., Coops, N. C., \& Wulder, M. A. (2015). Comparing ALS and Image-Based Point Cloud Metrics and Modelled Forest Inventory Attributes in a Complex Coastal Forest Environment. Forests, 6(10), 3704-3732.

White, J. C., Wulder, M. A., Vastaranta, M., Coops, N. C., Pitt, D., \& Woods, M. (2013). The utility of imagebased point clouds for forest inventory: A comparison with airborne laser scanning. Forests, 4(3), 518-536.

Yu, B., Gong, P., \& Pu, R. (1999). Penalized discriminant analysis of in situ hyperspectral data for conifer species recognition. Geoscience and Remote Sensing, IEEE Transactions on, 37(5), 2569-2577.

Yu, X., Hyyppä, J., Kukko, A., Maltamo, M., \& Kaartinen, H. (2006). Change detection techniques for canopy height growth measurements using airborne laser scanner data. Photogrammetric Engineering $\&$ Remote Sensing, 72(12), 1339-1348. 